\title{
ANÁLISE DO COMPORTAMENTO MORFOLÓGICO E RADIATIVO DE UM SISTEMA CONVECTIVO DE MESOESCALA INFERIDO VIA IMAGENS DE SATÉLITE
}

\author{
Luciana Barros Pinto ${ }^{1}$ e Cláudia Rejane Jacondino de Campos $^{2}$ \\ Recebido em 13 julho, 2006 / Aceito em 27 janeiro, 2009 \\ Received on July 13, 2006 / Accepted on January 27, 2009
}

\begin{abstract}
This article conducts a case study describing the radiational and morphological characteristics of a Mesoscale Convective System (MCS) responsible for the highest precipitations rates observed over Rio Grande do Sul State, Brazil, within the period spanning from November 2002 to February 2003. To that end, the FORecasting and TRAcking of the evolution of Cloud Clusters (ForTrACC) was used as the main tool. The selected case refers to the February 19 and 20, 2003 MCS event that caused high rainfall rates reported by the surface meteorological stations. This MCS displayed a life span of 23 hours, initiating at 13 UTC of February 19, reaching its mature stage at 04 UTC and dissipating at 11:30 UTC of the following day. During this entire period the MCS displayed cold tops with brightness temperatures below $210 \mathrm{~K}$. The behaviour of the MCS followed that described in typical conceptual models, where early strong convection is followed by lower values of brightness temperature, and then display area expansion in its mature stage. We also found distinct relations between minimum brightness temperatures obtained from satellite imagery and precipitation rates reported at the surface during the life cycle of the MCS. The conclusion is that the data collected from only two surface stations could not allow the determination of the clear-cut agreement between the radiative characteristics of the MCS observed by satellite and precipitation rates measured at the surface.
\end{abstract}

Keywords: Mesoscale Convective System, morphologic, brightness temperature, precipitation rates.

RESUMO. Nesse trabalho, foi feito um estudo de caso das características radiativas e morfológicas de um Sistema Convectivo de Mesoescala (SCM) causador dos maiores índices de precipitação observados sobre o Rio Grande do Sul, no período de novembro de 2002 a fevereiro de 2003, utilizando a ferramenta F0Recasting and TRAcking of the evolution of Cloud Clusters. 0 caso selecionado foi 0 dos dias 19 e 20/02/2003, onde apenas um sistema foi 0 responsável pela precipitação registrada nas estações meteorológicas em superfície. Esse sistema teve um ciclo de vida de 23 horas, com início às 13 UTC do dia 19/02/2003, maturação às 04 UTC e dissipação às 11:30 UTC do dia 20/02/2003. Durante todo esse período, apresentou topos frios com núcleos com temperatura abaixo de $210 \mathrm{~K}$. Ficou evidenciado que 0 sistema teve um comportamento semelhante ao descrito pelos modelos conceituais de SCMs, apresentando primeiramente forte convecção, associada a valores mais baixos de temperatura de brilho, para depois expandir-se no estágio de maturação. Os resultados mostraram a existência de diferentes padrões de relação entre a temperatura mínima de brilho e a precipitação registrada em superfície. Pode-se concluir que, com dados de apenas duas estações meteorológicas de superfície é difícil determinar se as características do SCM observado pelas imagens de satélite refletiram com confiabilidade o momento de maior atividade convectiva do SCM em superfície, caracterizado pelos maiores valores de precipitação.

Palavras-chave: Sistema Convectivo de Mesoescala, morfologia, temperatura de brilho, taxas de precipitação.

\footnotetext{
${ }^{1}$ Universidade Federal de Viçosa (UFV), Departamento de Engenharia Agrícola, 36570-000 Viçosa, MG, Brasil. Tel./Fax: +55 (31) 3899-1859 / (31) 3899-2735 -E-mail: luciana.meteoro@gmail.com

2Universidade Federal de Pelotas (UFPEL), Faculdade de Meteorologia, Campus Universitário, CP 354, 96010-900 Pelotas, RS, Brasil. Tel.: +55 (33) $3275-7329$ -E-mail: cjcampos@ufpel.edu.br
} 


\section{INTRODUÇÃo}

Os Sistemas Convectivos de Mesoescala (SCM) têm sido alvo de interesse de pesquisadores nas últimas décadas, devido às características de tempo a eles associados, como precipitações intensas, fortes rajadas de vento e granizo, que causam sérios danos à população.

Segundo Houze (1993), os SCMs possuem uma dinâmica mais complexa do que a das células convectivas individuais que os compõem. Segundo ele, o ciclo de vida dos SCMs pode ser dividido em quatro etapas. Durante 0 estágio de formação, o sistema surge como um grupo de células isoladas, ora distribuídas aleatoriamente, ora alinhadas. No estágio de desenvolvimento as células isoladas crescem e se fundem, com diversos núcleos de precipitação intensa, interligados por regiões de precipitação mais fraca. No estágio máximo, uma grande região de precipitação estratiforme é observada na retaguarda do SCM, e que persiste enquanto as novas células convectivas forem desenvolvidas na dianteira do sistema. E no estágio de dissipação, a formação de novas células diminui e o sistema se torna uma ampla região de precipitação estratiforme, enfraquecendo-se, restando apenas algumas células convectivas fracas. Esse comportamento também foi registrado por Machado \& Rossow (1993).

Diversos autores (Machado et al., 1994; Nicolini et al., 2002; Torres, 2003; Vila, 2004; Zipser et al., 2004) têm analisado vários SCMs com o objetivo de determinar 0 seu tamanho médio e seu horário preferencial de formação e de maturação. Esses estudos mostram que 0 tamanho médio dos sistemas está associado ao seu tempo de duração, com sistemas maiores apresentando maior duração. Com relação ao horário de formação dos sistemas, a maioria é detectada inicialmente no período da tarde - horário de máxima atividade convectiva no ciclo diurno sobre 0 continente - e um máximo secundário de formação é observado entre o fim da madrugada e 0 amanhecer (entre as 05 UTC e as 06 UTC). Também é observado que a fase madura dos sistemas tem dois picos, um pela tarde e outro durante a noite ou primeiras horas da manhã.

Outra característica observada é com relação à trajetória dos SCMs que se originam a leste da Cordilheira dos Andes, que segundo alguns autores (Guedes, 1985; Figueiredo \& Scolar, 1996; Nicolini et al., 2002; Torres, 2003) têm a tendência a se deslocarem para leste, entre $25^{\circ}$ e $40^{\circ} \mathrm{S}$.

Devido às muitas questões a serem ainda respondidas sobre os SCMs, o objetivo deste estudo é descrever, com o auxílio da ferramenta ForTrACC (FORecasting and TRAcking of the evolution of Cloud Clusters, Vila, 2004), o ciclo de vida e as propriedades morfológicas e radiativas do SCM causador do evento de maiores taxas de precipitação, ocorrido sobre o Rio Grande do Sul, durante o período que integra o experimento SALLJEX (South American Low-Level Jet Experiment). 0 experimento SALLJEX, que visa uma melhor compreensão dos Jatos de Baixos Níveis na América do Sul, ocorreu de novembro de 2002 a fevereiro de 2003, abrangendo a faixa de $40^{\circ}$ a $0^{\circ} \mathrm{Se} 85^{\circ}$ a $35^{\circ} \mathrm{W}$.

\section{MATERIAL E MÉTODOS}

Neste trabalho foram utilizadas as imagens do satélite Geostationary Operational Environmental Satellite (GOES $8-75^{\circ} \mathrm{W}, 0^{\circ}$ ) com uma resolução espacial de $4 \mathrm{~km} \times 4 \mathrm{~km}$ e resolução temporal de 1/2 hora, correspondentes ao canal $4(10,7 \mu \mathrm{m}$, infravermelho térmico), disponibilizadas via anonymous FTP pelo National Centers for Environmental Prediction (NCEP). Em conjunto, utilizouse dados observados de precipitação acumulada da 00 UTC Coordenada de Tempo Universal (total acumulado em 6 horas), 12 UTC (total acumulado em 12 horas) e 18 UTC (total acumulado em 6 horas), assim como a precipitação total acumulada no período de estudo, a partir de 22 estações meteorológicas de superfície (8 DISME-INMET), incluindo a análise de pluviogramas. 0 período examinado para a seleção do evento convectivo foi de novembro de 2002 a fevereiro de 2003 e a região de interesse foi 0 estado do Rio Grande do Sul (RS).

Primeiramente, analisando os dados de precipitação, foi selecionado 0 dia com a maior precipitação em superfície registrada na maioria das estações meteorológicas. Então, foram analisadas as imagens de satélite correspondentes, a fim de se identificar a existência de algum tipo de SCM sobre o RS que pudesse ter gerado essa precipitação.

Após a análise objetiva (manual) das imagens de satélite, foi utilizada a versão diagnóstica da ferramenta ForTrACC para um melhor detalhamento do ciclo de vida e das características morfológicas e radiativas do(s) sistema(s) convectivo(s) que desenvolveu-se(desenvolveram-se) sobre a região e que foi(foram) responsável(eis) pela precipitação registrada em superfície.

Como descrito por Vila et al. (2004), o ForTrACC é um método automático para o monitoramento e documentação dos SCMs através do seu ciclo de vida, utilizando informações dos canais térmicos dos satélites geoestacionários. Os principais elementos que compõem este método são: 1) um algoritmo de detecção e agrupamento ('clusters') usando um limiar de temperatura; 2) um programa para 0 cálculo dos diversos parâmetros estatísticos e morfológicos dos sistemas encontrados na etapa anterior (Machado et al., 1998; Vila \& Machado, 2003); 3) uma técnica de acompanhamento em si, baseada na superposição de áreas entre 
imagens sucessivas, o que permite a construção das trajetórias dos sistemas ao longo de seu ciclo de vida (famílias) (Mathon \& Laurent, 2001; Laurent et al., 2002) e 4) uma etapa de geração de imagens virtuais ou sintéticas, utilizadas para cobrir os horários de imagens faltantes (esta última etapa não foi necessária neste estudo). Neste trabalho foi utilizado o limiar de temperatura de brilho de $235 \mathrm{~K}$, para definir o SCM, e o de $210 \mathrm{~K}$, para definir as células convectivas mais intensas imersas no SCM, que são os valores utilizados pelo ForTrACC (Vila, 2004).

Entre as etapas descritas acima, uma das principais é a da formação das "famílias". Nessa etapa, o programa acompanha os sistemas desde o seu surgimento até a sua dissipação, levando em consideração todas as fusões e divisões sofridas por ele ao longo do seu ciclo de vida e, a esse conjunto dá-se o nome de família (que é utilizado para designar "cluster"). Assim, família é o comportamento dos sistemas ao longo da sua trajetória durante todo o ciclo de vida. Neste trabalho uma família será denominada de sistema, ou SCM.

Para a seleção do caso, procurou-se identificar, para cada estação meteorológica de superfície do RS, se um SCM deslocouse sobre as mesmas entre as 12 UTC do dia 19/02/03 e as 12 UTC do dia 20/02/03. Para isso, foi utilizado um programa que confronta o conjunto de sistemas gerado pelo ForTrACC com os dados de latitude e longitude de cada estação.

Uma vez cumprida a etapa de seleção descrita acima, pôdese estudar 0 ciclo de vida, a trajetória e as características morfológicas e radiativas do sistema.

Por fim, os dados de precipitação das estações de superfície, que tiveram precipitação acima de $75 \mathrm{~mm}$ (acumulada em 24 horas) e os dados de temperatura mínima (temperatura de briIho mínima, estimada via satélite), gerados pelo ForTrACC, foram confrontados. Este confronto serviu para verificar a existência de um padrão de relação entre as características do SCM observado pelas imagens de satélite e o que ocorreu em superfície.

\section{RESULTADOS E DISCUSSÃO}

0 caso selecionado foi o ocorrido durante os dias 19 e 20 de fevereiro de 2003, onde em algumas estações de superfície foram registrados valores de precipitação (acumulada em 24 horas) acima de 75 mm, às 12 UTC do dia 20/02/03.

A Figura 1 mostra a precipitação acumulada entre as 12 UTC do dia 19/02/03 e as 12 UTC do 20/02/03, para 22 estações meteorológicas de superfície do Rio Grande do Sul. Pode-se observar nesta figura, que os maiores valores de precipitação foram registrados no centro e norte do RS, com um núcleo de valores mais intensos (acima de $100 \mathrm{~mm}$ ) no nordeste do estado.
Como 0 interesse deste trabalho é 0 estudo do caso de precipitação intensa registrado às 12 UTC do dia 20/02/03, optouse por analisar somente os sistemas convectivos que atuaram sobre 0 RS, das 12 UTC do dia 19/02/03 às 12 UTC do dia 20/02/03 (24 horas), visto que no dia anterior não houve registro de precipitação. Assim, os resultados aqui apresentados utilizando a ferramenta ForTrACC, referem-se a este período.

Pela análise das imagens de satélite (Fig. 2) pode-se observar que 0 sistema surgiu da dissipação de uma linha de instabilidade às 13 UTC (Fig. 2a), cortando o estado do RS de norte a sul e deslocando-se para leste. Essa linha de instabilidade formou-se na região anterior a uma frente fria que se deslocou do Uruguai para o Oceano Atlântico, sem passar pelo RS. Às 16:30 UTC (Fig. 2b) surgiram novos núcleos convectivos a oeste do sistema principal, que acabaram unindo-se ao mesmo às 18 UTC (Fig. 2C), fazendo com que o deslocamento do centro de massa desse novo sistema fosse para norte. 0 mesmo aconteceu às 20 UTC (Fig. 2d), quando o sistema que estava no nordeste do estado fundiu-se com novos núcleos convectivos que surgiram na divisa noroeste com a Argentina e no sul do Paraguai. 0 desenvolvimento e a maturação do sistema foram alimentados por um jato de baixos níveis que manteve a convecção no norte do RS (Pinto \& Campos, 2007). A partir deste momento, 0 sistema ficou estacionado sobre o norte do estado, apresentando às 04 UTC (Fig. 2f) a maior extensão coberta por topos frios (com temperatura de brilho abaixo de $210 \mathrm{~K}$ ) e, alcançando às 07:30 UTC (Fig. 2g) sua maior extensão. A partir deste horário, o sistema começou a enfraquecer e dissipar-se, até deixar o RS às 11:30 UTC (Fig. 2h). É importante notar que a descrição da evolução do sistema através das imagens de satélite, aqui apresentada, é semelhante à proposta por Houze (1993) e por Machado \& Rossow (1993), conforme descrito na introdução deste trabalho. Notou-se também, que devido ao fato de o deslocamento do sistema ser determinado pelo seu centro de massa, a trajetória deste foi errática, pois seu centro de massa mudou constantemente a cada fusão ou separação sofrida pelos sistemas que a compunham.

Observou-se que um mesmo SCM atuou sobre o RS durante 23 horas, tendo início às 13 UTC do dia 19/02/03 e fim às 12 UTC do dia 20/02/03. Esse sistema foi o único responsável pela precipitação registrada em superfície.

Para uma melhor descrição deste SCM, têm-se as Figuras $3 \mathrm{e}$ 4, que mostram a evolução de alguns de seus parâmetros físicos e radiativos ao longo do seu ciclo de vida.

Analisando em conjunto as Figuras 3 e 4 pode-se notar que a partir do início do acompanhamento do SCM às 13 UTC até 


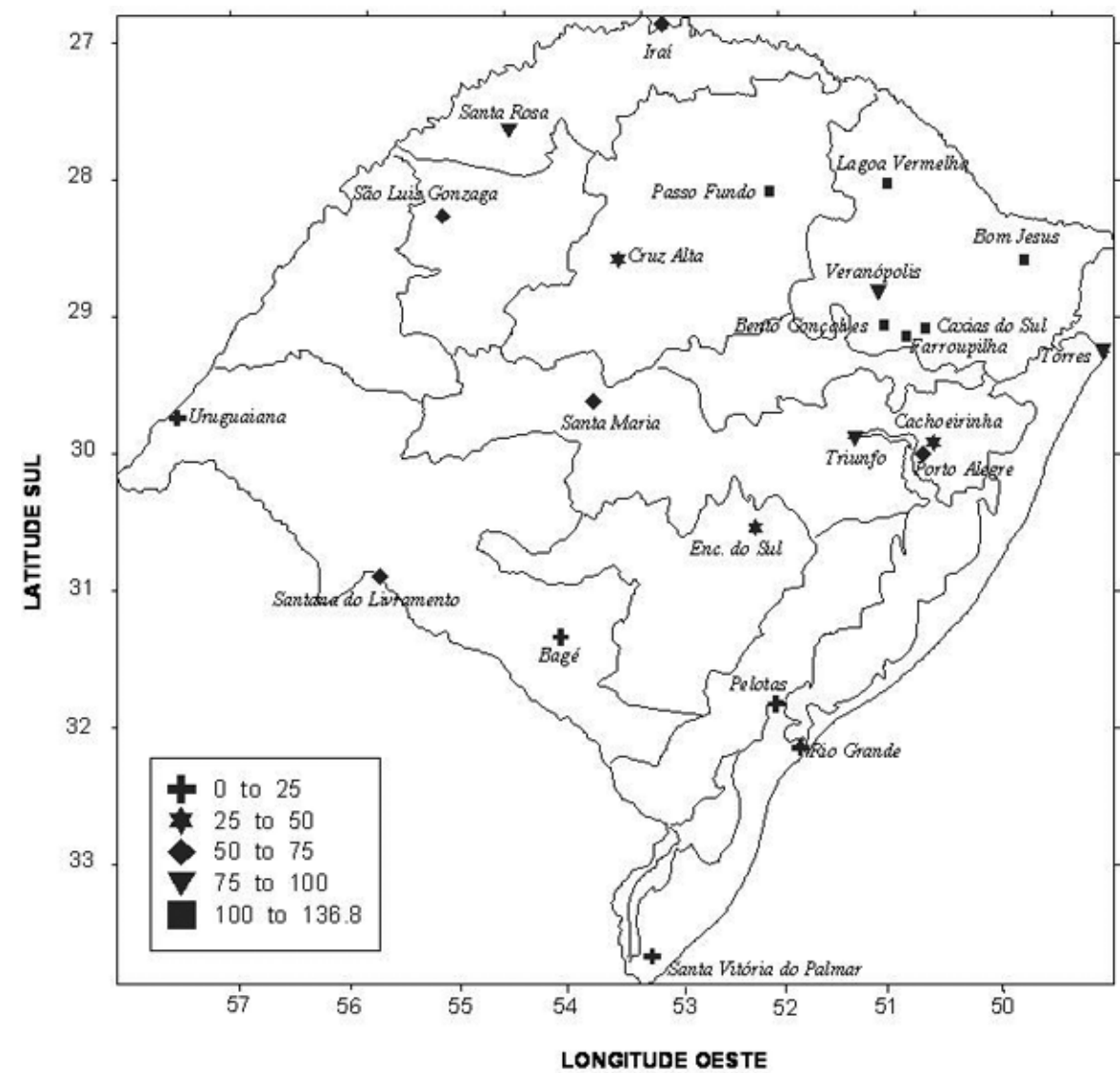

Figura 1 - Precipitação acumulada (mm) das 12 UTC do dia 19/02/03 às 12 UTC do dia 20/02/03, para 22 estações meteorológicas de superfície do Rio Grande do Sul.
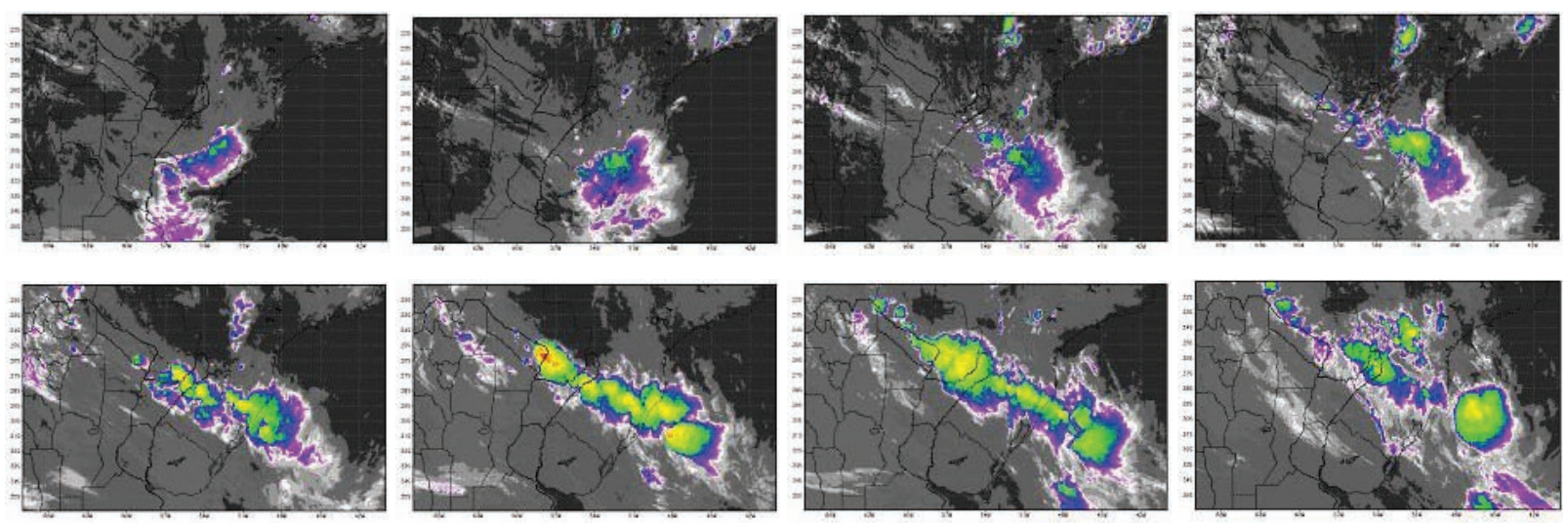

Figura 2 - Imagens do satélite GOES 8, no canal infravermelho térmico (NCEP), mostrando a evolução do SCM entre os dias 19 e 20 de fevereiro de 2003.

as 23 UTC do dia 19/02/03, este apresentou um lento aumento de tamanho, acompanhado de uma queda suave nos valores de temperatura de brilho média e mínima (abaixo de 235 e $210 \mathrm{~K}$, respectivamente). 0 tamanho do sistema é dado pelo número de pixels com temperatura de brilho inferior a $235 \mathrm{~K}$. 0 método de identificação de um mesmo SC no tempo "t" e nas imagens sucessivas em " $\mathrm{t}+\Delta \mathrm{t}$ " é baseado no critério de máxima superposição da área dos SC em imagens sucessivas. É adotado um número 


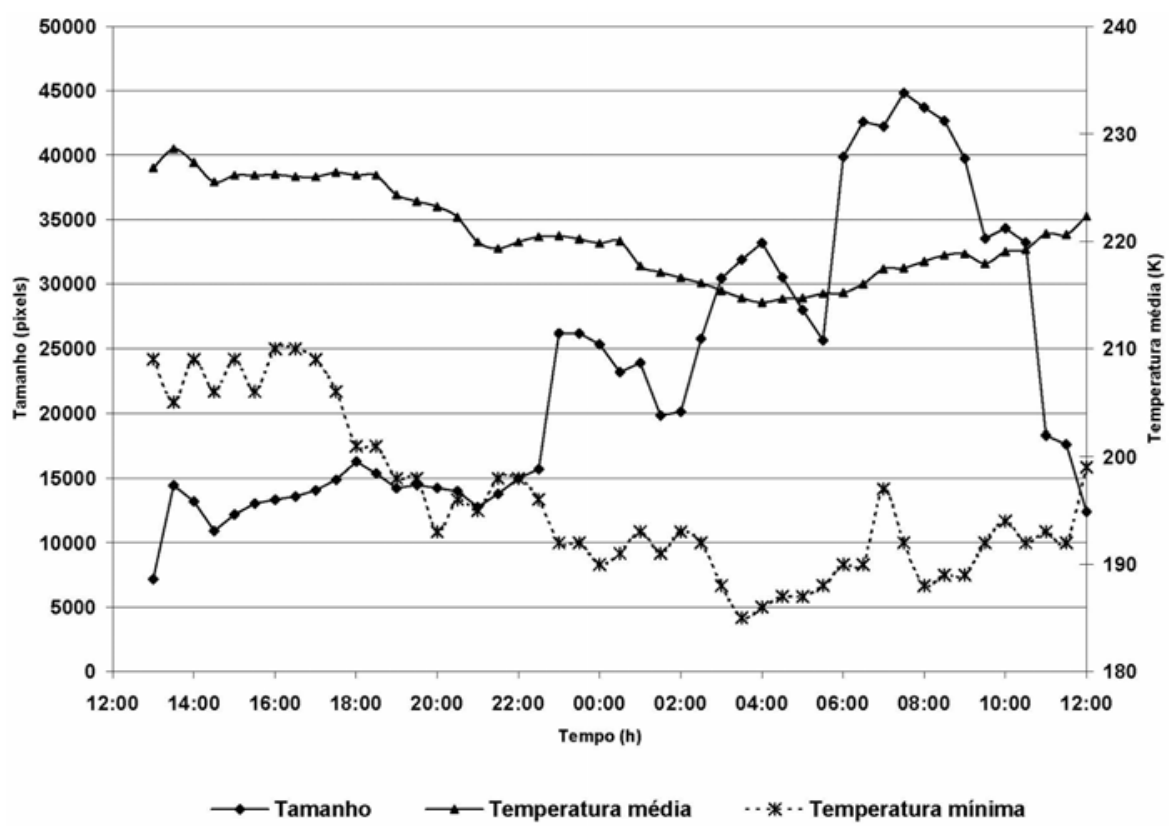

Figura 3 - Evolução temporal do tamanho (em número de pixels com temperatura abaixo de $235 \mathrm{~K}$ ); da temperatura de brilho média (abaixo de $235 \mathrm{~K}$ ) e da temperatura de brilho mínima (abaixo de $210 \mathrm{~K}$ ) do SCM que atingiu o estado do Rio Grande do Sul entre as 12 UTC do dia 19/02/03 e as 12 UTC do dia 20/02/03.

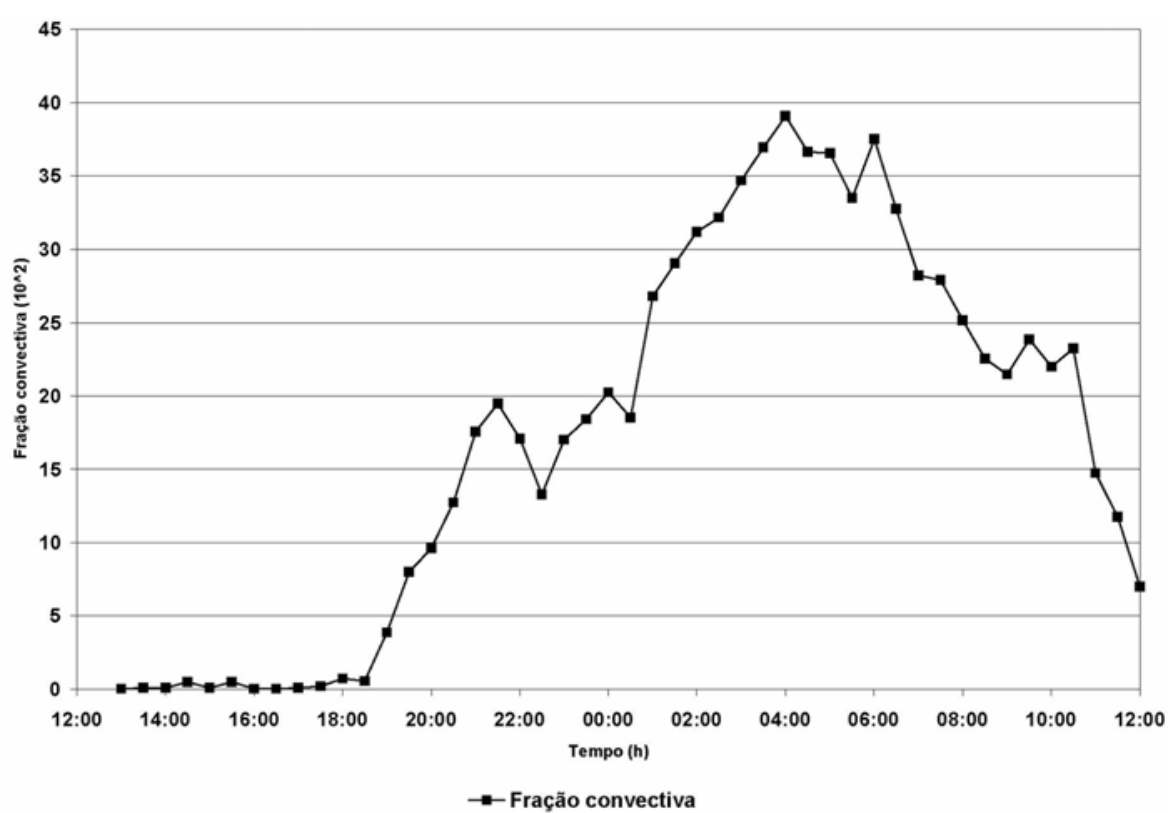

Figura 4 - Evolução temporal da fração convectiva (estimada via satélite) do SCM que atingiu o estado do Rio Grande do Sul, entre as 12 UTC do dia 19/02/03 e as 12 UTC do dia 20/02/03.

mínimo de pixels para considerar a continuidade do SC, atualmente de 150 pixels (aproximadamente $2400 \mathrm{~km}^{2}$ ) para um $\Delta t$ = 30 minutos. Se a superposição for menor, 0 sistema não é considerado como sendo o mesmo sistema no tempo anterior (Vila, 2004).
A partir das 22:30 UTC do dia 19/02/03, o sistema começou a expandir-se de forma mais significativa, até atingir sua máxima área às 7:30 UTC do dia 20/02/03 (44822 número de pixels, com temperatura de brilho inferior a $235 \mathrm{~K}$, aproximadamente 717.152 $\mathrm{km}^{2}$ ). Embora sua maior área tenha sido às 7:30 UTC, 0 horário 
de maior atividade convectiva do sistema foi às 04 UTC, quando 0 sistema atingiu menores valores de temperatura de brilho média e mínima, e maior valor da fração convectiva. Como ilustrado na Figura 4, das 18 UTC do dia 19/02/03 às 04 UTC do dia 20/02/03 o sistema sofreu um aumento na sua fração convectiva, que é a relação entre a área do sistema abaixo de $210 \mathrm{~K}$ e a área total do SCM (pixels com temperatura de brilho inferior a $235 \mathrm{~K}$ ). Isso evidencia o horário em que o SCM atingiu seu período com convecção mais intensa.

Após atingir sua maior extensão, o sistema começou a dissipar-se, aumentando as temperaturas de brilho média e mínima e diminuindo a fração convectiva.

0 mais interessante dessa análise é observar que 0 sistema primeiro sofreu uma diminuição da temperatura mínima de brilho (às 3:30 UTC do dia 20/02/03), para depois alterar a temperatura média de brilho (com mínimo às 04 UTC do dia 20/02/03) e a fração convectiva, e só depois então, expandiu-se até atingir sua maior área. Isso confirma a teoria, descrita por Machado \& Rossow (1993) e também observada por Vila (2004), de que durante a etapa de formação, o sistema alcança um máximo resfriamento e logo se expande na etapa de maturação.

Em seguida, para verificar a existência de um padrão de relação entre as características do SCM observado pelas imagens de satélite e 0 que ocorreu em superfície foram estudadas algumas das estações que tiveram precipitação acima de $75 \mathrm{~mm}$ (acumulada em 24 horas). Analisou-se o comportamento da precipitação registrada em superfície e da temperatura de brilho mínima (estimadas via satélite) para o pixel do sistema melhor localizado sobre a estação estudada.

A Figura 5 mostra 0 comportamento da temperatura de briIho mínima para os pixels posicionados sobre as estações meteorológicas de Lagoa Vermelha e Caxias do Sul. Essas estações foram escolhidas por:

(i) estarem localizadas ao longo da área de maior atividade do sistema; e

(ii) representarem localidades que sofreram simultaneamente a ação do SCM.

Na Figura 5, as falhas no gráfico são referentes a horários em que a temperatura de brilho para 0 pixel localizado sobre a estação correspondente esteve acima de $235 \mathrm{~K}$.

Uma característica observada pela análise da Figura 5, é que o comportamento da temperatura mínima de brilho sobre as estações é semelhante ao comportamento do sistema, ou seja, os mínimos de temperatura mínima de brilho sobre as estações coincidem com o horário de menor temperatura média de brilho do sistema, mostrando que essas estações estão localizadas no centro convectivo do sistema.

Com relação à precipitação registrada em superfície pode-se notar pela Tabela 1, para as duas cidades que os seus valores foram mais significativos no horário das 12 UTC do dia 20/02/03 (acumulado da 00 UTC do dia 20/02/03 até as 12 UTC do dia 20/02/03). Para Caxias do Sul a precipitação registrada em superfície começou a ser mais significativa no horário da 00 UTC, que corresponde ao valor acumulado precipitado entre as 18 UTC do dia 19/02/03 e a 00 UTC do dia 20/02/03.

Tabela 1 - Precipitação acumulada $(\mathrm{mm})$ registrada nas estações de superfície de Lagoa Vermelha e Caxias do Sul.

\begin{tabular}{|c|c|c|c|c|}
\hline \multirow{2}{*}{$\begin{array}{c}\text { Estações } \\
\text { meteorológicas }\end{array}$} & \multicolumn{4}{|c|}{ Precipitação (mm) } \\
\cline { 2 - 5 } & $\begin{array}{c}\text { 18 UTC } \\
(19 / 02)\end{array}$ & $\begin{array}{c}\text { 00 UTC } \\
(20 / 02)\end{array}$ & $\begin{array}{c}12 \text { UTC } \\
(20 / 02)\end{array}$ & Total \\
\hline Lagoa Vermelha & 0,6 & 7,4 & 97,2 & 105,2 \\
\hline Caxias do Sul & 8,4 & 59,2 & 69,1 & 136,7 \\
\hline
\end{tabular}

A análise da Figura 5 e dos registros dos pluviogramas das estações meteorológicas de Lagoa Vermelha e Caxias do Sul (não apresentados aqui) mostram que não é possível determinar uma relação direta ou inversa entre a temperatura mínima de brilho e a precipitação em superfície, analisando apenas estas duas cidades. Percebem-se diferentes padrões de relação entre essas duas variáveis. Isso pode ser confirmado quando analisamos, por exemplo, o período de precipitação mais significativa. Observa-se, neste período uma relação inversa entre essas variáveis, ou seja, em Caxias do Sul, no período da 00 UTC às 04 UTC do dia 20/02/03 e em Lagoa Vermelha, no período das 04 UTC às 07 UTC desse mesmo dia, onde foi registrado um mínimo de temperatura mínima de brilho (ver Fig. 5), para as duas cidades, a precipitação registrada em superfície foi elevada, respectivamente, $52 \mathrm{~mm}$ (aproximadamente 40\% do total de precipitação registrada em superfície, no período entre as 12 UTC do dia 19/02 e as 12 UTC do dia 20/02) e $70 \mathrm{~mm}$ (aproximadamente 70\% da precipitação total registrada em superfície, no período entre as 12 UTC do dia 19/02 e as 12 UTC do dia 20/02).

Por outro lado, quando essa mesma análise é feita para outros períodos, observa-se que não se mantém a mesma relação entre temperatura mínima de brilho e precipitação em superfície. Podem-se citar como contra-exemplos, duas outras situações. A primeira para Caxias do Sul, que no período compreendido entre as 18 UTC e as 21 UTC do dia 19/02/03, registrou $50 \mathrm{~mm}$ de precipitação em superfície, de acordo com o seu pluviograma, e temperatura mínima de brilho (Fig. 5) acima de $235 \mathrm{~K}$. 


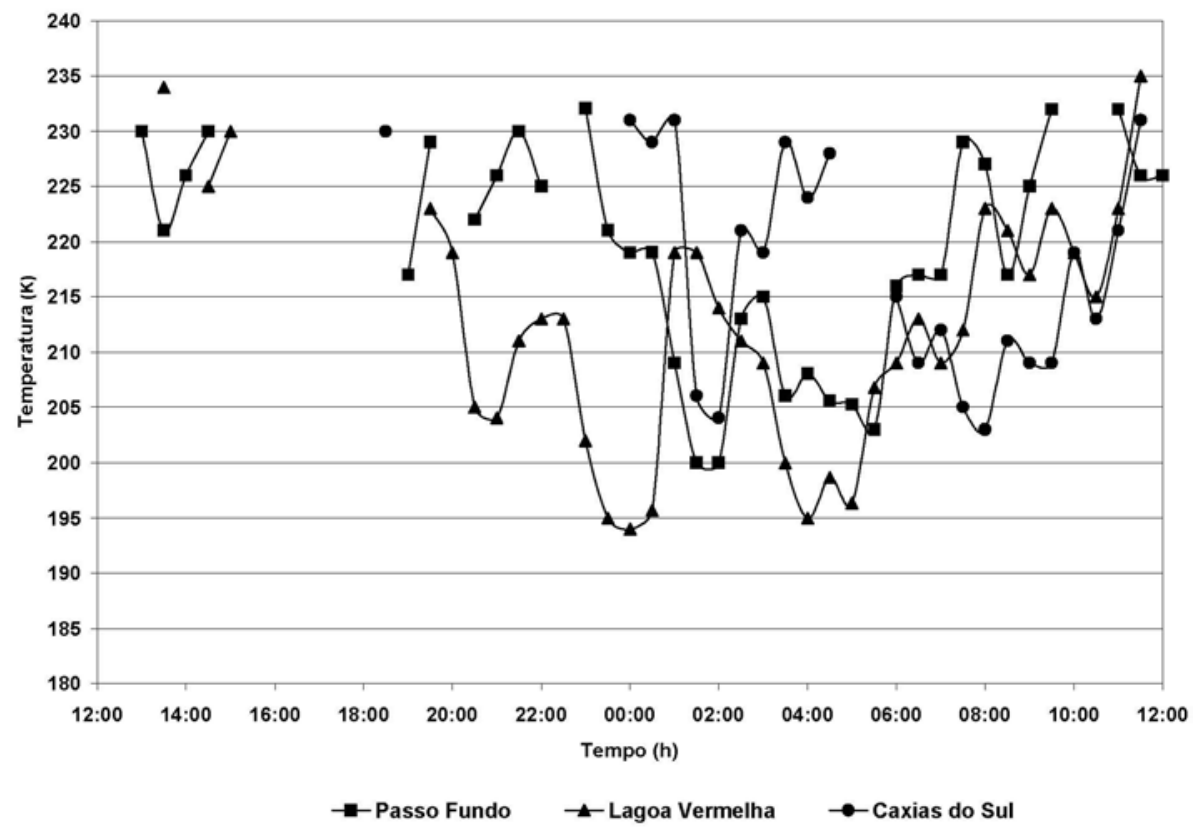

Figura 5 - Evolução temporal da temperatura de brilho mínima (K) para os pixels localizados sobre as estações meteorológicas de superfície de Lagoa Vermelha e Caxias do Sul, entre as 12 UTC do dia 19/02/03 e as 12 UTC do dia 20/02/03. Apenas valores de temperatura de brilho abaixo de $235 \mathrm{~K}$ estão plotados.

A análise das duas variáveis mostra um comportamento diverso daquele do período da 00 UTC às 04 UTC, ou seja, nesta situação tem-se uma relação direta entre as duas variáveis, com precipitação intensa num período onde a temperatura mínima de brilho encontra-se elevada. A segunda situação analisada foi para Lagoa Vermelha, que no período das 23 UTC do dia 19/02/03 à 01 UTC do dia 20/02/03, registrou 14 mm de precipitação em superfície, de acordo com o seu pluviograma, e um mínimo de temperatura mínima de brilho (Fig. 5). Nesta situação tem-se uma relação direta entre as duas variáveis, com precipitação relativamente fraca num período onde ocorreu um mínimo de temperatura mínima de brilho.

Com base nos resultados apresentados acima não foi possível evidenciar a relação inversa entre temperatura mínima de brilho e taxa de precipitação (discutida em Vila, 2004) durante toda a evolução do SCM. Dois contra-exemplos foram encontrados: um onde precipitação intensa ocorre quando a temperatura de brilho encontra-se elevada e outro onde a precipitação fraca ocorre durante um mínimo de temperatura de brilho. Entretanto, é evidente que mais casos devem ser estudados - envolvendo um número maior de estações meteorológicas de superfície - para se verificar o grau de generalidade desta conclusão no contexto de significância estatística. Estes estudos adicionais devem também levar em consideração processos atmostéricos não examinados aqui, como por exemplo, fatores que influenciam a eficiência da precipitação no SCM.

\section{CONCLUSÃO}

Um SCM ocorrido sobre o Rio Grande do Sul durante o período referente ao experimento SALLJEX (novembro de 2002 a fevereiro de 2003) foi selecionado para estudo utilizando-se como critério a precipitação total acumulada em superfície. Este SCM, ocorrido entre os dias 19 e 20 de fevereiro de 2003, foi responsável pelas mais altas taxas de precipitação registradas nas estações meteorológicas daquele estado durante o período em questão.

Esse sistema teve um ciclo de vida de 23 horas, com início às 13 UTC do dia 19/02/03, maturação às 04 UTC e dissipação às 11:30 UTC do dia 20/02/03. Durante todo esse período, apresentou topos frios, ou seja, núcleos com temperatura abaixo 210 K. Ficou evidenciado também que 0 sistema teve um comportamento semelhante aos modelos conceituais de evolução de SCMs, primeiro apresentando uma intensa convecção acompanhada de queda de temperatura de brilho no seu topo, para depois se expandir no estágio de maturação. 0 sistema também mostrou que 0 seu período de maturação coincide com o citado na literatura, ou seja, um pico bem marcado durante o final da madrugada e início da manhã. Devido ao fato deste sistema ter sido marcado por várias fusões e separações durante seu ciclo de vida, sua 
trajetória foi aleatória.

Para 0 estudo de caso apresentado neste trabalho, não foi possível caracterizar um padrão único de relação entre a temperatura de brilho e a precipitação em superfície. Foram observadas três situações diferentes: uma com precipitação intensa num período no qual ocorreu um mínimo de temperatura mínima de brilho; outra com precipitação intensa num período cuja temperatura mínima de brilho encontra-se elevada e outra com precipitação fraca num período onde ocorreu um mínimo de temperatura mínima de brilho. A análise dos dados de apenas duas estações não permitiu determinar se as características do sistema observado pelas imagens de satélite refletem com confiabilidade o momento de ocorrência de maior precipitação em superfície. Um estudo mais detalhado utilizando mais dados de registro de precipitação ao longo da área de atuação do sistema é necessário para se confirmar esses resultados.

\section{REFERÊNCIAS}

FIGUEIREDO JC \& SCOLAR J. 1996. Estudo da trajetória dos sistemas convectivos de mesoescala na América do Sul. In: VII Congresso Argentino de Meteorologia e VII Congresso Latino-Americano e Ibérico de Meteorologia, Buenos Aires, Argentina, Anais, 165-166.

GUEDES RL. 1985. Condições de grande escala associadas a sistemas convectivos de mesoescala sobre a região central da América do Sul. Dissertação de Mestrado, IAG/USP, 89 pp.

HOUZE RA.1993. Mesoscale convective systems. In: HOUZE RA. Cloud dynamics. Academic Press, Inc., 53: 334-404.

LAURENT H, MACHADO LAT, MORALES C \& DURIEUX L. 2002. Characteristics of Amazonian Mesoscale Convective Systems observed from Satellite and radar during the WETAMC/LBA Experiment, J. Geophys. Res., 107(D20): 8054.

MACHADO LAT \& ROSSOW WB. 1993. Structural radiative and characteristics of tropical cloud clusters. Mon. Wea. Rev., 121(12): 3234-3260.
MACHADO LAT, GUEDES RL, SILVEIRA JMB, WALTZ RC \& ALVES MAS. 1994. Ciclo de vida de sistemas convectivos. In: VIII Congresso Brasileiro de Meteorologia, SBMET, Anais, 2: 323-326.

MACHADO LAT, ROSSOW WB, GUEDES RL \& WALKER AW. 1998. Life cycle variations of mesoscale convective systems over the Americas. Mon. Wea. Rev., 126: 1630-1654.

MATHON V \& LAURENT H. 2001. Life cycle of the Sahelian mesoscale convective cloud systems. Quart. J. Roy. Meteo. Soc., 127: 377-406.

NICOLINI M, SAULO AC, TORRES JC \& SALIO P. 2002. Enhanced precipitation over Southeastern South America related to strong low-level jet events during austral warm season. Meteorologica, Special Issue for the South American Monsoon System, 27: 59-69.

PINTO LB \& CAMPOS CRJ. 2007. Ambiente sinótico e perfis verticais de um Sistema Convectivo de Mesoescala (SCM). Revista Brasileira de Geofísica, 25(1): 31-43.

TORRES JC. 2003. Sistemas Convectivos en Mesoescala Altamente Precipitantes en el Norte y Centro de Argentina. Tesis de Doctorado. FCEyN-UBA, 130 pp.

VILA DA. 2004. Sistemas Convectivos Precipitantes de Mesoescala sobre Sudamerica: ciclos de vida y circulación en gran escala asociada. Tesis de Doctorado. FCEyN-UBA, 126 pp.

VILA DA \& MACHADO LAT. 2004. Shape and Radiative Properties of Convective Systems observed from Infrared Satellite Images. International Journal of Remote Sensing, 25(21): 4441-4456.

VILA DA, MACHADO LAT, VELASCO I \& MACEDO SR. 2004. LOS sistemas convectivos de mesoescala sobre la cuenca Del Plata: La Técnica FORTRACC. In: XIII Congresso Brasileiro de Meteorologia, CD-ROM do XIII CBMet.

ZIPSER EJ, SALIO P \& NICOLINI M. 2004. Mesoscale convective systems activity during SALLJEX and the relationship with SALLJ events. CLIVAR Exchanges, 9(1): 14-18.

\section{NOTAS SOBRE OS AUTORES}

Luciana Barros Pinto. Doutoranda do curso de Meteorologia Agrícola, pela Universidade Federal de Viçosa - UFV. Foi professora substituta do Departamento de Geociências da Fundação Universidade Federal do Rio Grande (2006-2007). Graduação em Meteorologia pela Universidade Federal de Pelotas, UFPEL (2000-2003). Mestrado em Meteorologia pela UFPEL (2004-2006). Áreas de interesse: Meteorologia, meteorologia de mesoescala, meteorologia sinótica, modelagem climática e mudanças climáticas.

Claudia Rejane Jacondino de Campos. Professora associada do Departamento de Meteorologia, da Faculdade de Meteorologia da Universidade Federal de Pelotas. Graduação em Meteorologia, pela Universidade Federal de Pelotas, UFPEL (1984-1987), Mestrado em Agronomia (Meteorologia Agrícola) pela Universidade Federal de Viçosa, UFV (1988-1990) e Doutorado em Física da Atmosfera, pela Université de Toulouse III (Paul Sabatier), U.T. III, França (1993-1996). Áreas de interesse: Meteorologia, micrometeorologia, meteorologia de mesoescala, meteorologia sinótica e agrometeorologia. 